\title{
30. DATA REPORT: CHEMICAL AND ISOTOPIC COMPOSITIONS OF PORE FLUIDS IN SEDIMENTS OF THE CASCADIA ACCRETIONARY COMPLEX'
}

\author{
Miriam Kastner ${ }^{2}$ and Henry Elderfield ${ }^{3}$
}

\begin{abstract}
To complement the shipboard pore fluid chemical data from the Cascadia accretionary complex, Sites 888 to 892 , the chemical and isotopic data reported here were carried out in our shore-based laboratories. The data include: (1) lithium and strontium concentrations and strontium and oxygen isotope ratios for all sites drilled, both off Vancouver Island and off central Oregon; (2) calcium, magnesium, potassium, and sodium concentrations for Hole 892D pore fluid samples; and (3) strontium and oxygen isotope ratios of a pure massive gas hydrate sample from Hole 892D. Shipboard $\mathrm{Cl}$ concentrations are included for better sample identification. The data reflect fluid-rock reactions and, at least at Site 892, advection of fluid from deeper in the accretionary complex.
\end{abstract}

\section{INTRODUCTION}

The existence of large-scale fluid flow and fluid expulsion at convergent margins is demonstrated by (1) tectonically induced rapid porosity reduction of accretionary complexes; (2) regionally variable heat flow; (3) pore-fluid chemical, isotopic, and temperature anomalies that can only be maintained by rapid fluid flow; and (4) widespread diffuse and/or channelized fluid venting through mud volcanoes or along thrusts. These fluids sustain prolific benthic biological communities and cause carbonate deposition, primarily from methane oxidation. High pore fluid pressures are pervasive in convergent margins. These fluids play a central role in the deformational, thermal, and chemical evolution of convergent margins, and enhance diagenetic and metamorphic reactions. Fluids released from these reactions may be important for global geochemical budgets; $\mathrm{H}_{2} \mathrm{O}$ and $\mathrm{CO}_{2}$ may expedite partial melting at greater depths.

The expelled fluids are characterized by variable chemical and isotopic compositions. Fresher-than-seawater fluids are ubiquitous in accretionary complexes; seawater dilutions of $10 \%-64 \%$ have been recorded. In the Cascadia accretionary complex a maximum seawater dilution of $37 \%$ was observed at Site 889 , and a $22 \%$ seawater dilution was recorded in Hole 892D. Mostly gas hydrate dissociation contributes to the observed $\mathrm{Cl}$ dilution at both sites.

Deciphering the origin(s) of the geochemically complex pore fluids in sediments of accretionary complexes requires an array of chemical and isotopic data. Accordingly, the geochemical and isotopic data reported here provide new insights into the origin and flow of fluids in the Cascadia accretionary complex.

\section{MATERIALS AND METHODS}

All pore fluids analyzed were obtained by routine shipboard squeezing of whole-round sediment samples immediately after retrieval. The sediments were squeezed in titanium squeezers at ambient temperature and at pressures of $135-205 \mathrm{MPa}\left(140-210 \mathrm{~kg} / \mathrm{cm}^{2}\right)$.

'Carson, B., Westbrook, G.K., Musgrave, R.J., and Suess, E. (Eds.). 1995. Proc ODP, Sci. Results, 146 (Pt. 1): College Station, TX (Ocean Drilling Program).

${ }^{2}$ Scripps Institution of Oceanography, La Jolla, CA 92093-0212, U.S.A.

'Department of Earth Sciences, University of Cambridge, Cambridge CB2 3EQ. United Kingdom.
The pore fluid samples were analyzed on board ship for a range of constituents. The shore-based pore fluid analyses emphasized lithium and strontium concentrations and strontium and oxygen isotope ratios, summarized in Tables 1 through 9. Because of some shipboard problems with the atomic absorption spectrophotometer, all pore fluid samples were analyzed for $\mathrm{Li}$ concentrations at the Scripps Institution of Oceanography (SIO) laboratories, including the samples from the three holes $(889 \mathrm{~A}, 891 \mathrm{~A}$, and $891 \mathrm{~B})$ analyzed for $\mathrm{Li}$ on board ship. Pore fluids from Hole 892D were also analyzed for $\mathrm{Ca}, \mathrm{Mg}, \mathrm{K}$, and $\mathrm{Na}$ concentrations using the shipboard methods described in Gieskes et al. (1991). Lithium and strontium concentrations were measured by atomic absorption spectroscopy. The precision for $\mathrm{Sr}$ was $<3.0 \%$ and for Li only $10 \%$ because of the low $\mathrm{Li}$ concentrations. Strontium and oxygen isotope ratios were determined by mass spectrometry. For the pore fluid oxygen isotope analysis, the method of Bottinga and Craig (1969) was used with a precision of $0.08 \%$. Strontium isotopes were determined by the previously described method (e.g., Elderfield and Gieskes, 1982). The $2 \sigma$ errors are given in Tables 6 through 9. Measured ${ }^{87} \mathrm{Sr} /{ }^{86} \mathrm{Sr}$ ratios were normalized to a particular value (to ${ }^{86} \mathrm{Sr}{ }^{88} \mathrm{Sr}=0.1194$ ) for a standard; the value obtained for the National Bureau of Standards (NBS) 987 standard measured in the SIO laboratory is 0.710255 , with a $2 \sigma$ error of 18 .

\section{GENERAL RESULTS}

The data indicate the uniqueness of Site 892 pore-fluid geochemistry relative to the other drilled sites on Leg 146 at the Cascadia accretionary complex. At Site 892, the Li and $\mathrm{Sr}$ concentrations are two to three times higher (Figs. 1,2) and the ${ }^{87} \mathrm{Sr} /{ }^{86} \mathrm{Sr}$ significantly less radiogenic (Fig. 3). The pore-fluid $\mathrm{Sr}$ isotope data of all sites are less radiogenic than modern or contemporary seawater $\mathrm{Sr}$ isotopic values (e.g., Burke et al., 1982; see Tables 6-9 and Fig. 3). The slightly negative oxygen isotope values in Tables $6-9$ and Figure 4 reflect in-situ low-temperature formation of diagenetic minerals of either carbonates and/or silicates.

\section{GAS HYDRATE}

Gas hydrate was recovered between 2 and 5 and $\sim 19$ mbsf in Holes 892A, 892D, and 892E. Most of it occurs as individual, dis- 
persed, platy-to-prismatic 1- to 1.5 -cm-long white crystals or in crystal clusters. Only one 2 - to 3 -cm-thick massive gas hydrate layer was obtained in Core 146-892D-3X; it was immediately frozen in liquid nitrogen. This is a $\mathrm{CH}_{4}-\mathrm{H}_{2} \mathrm{~S}$ gas hydrate, with $\sim 10 \% \mathrm{H}_{2} \mathrm{~S}$; it also contains minor amounts of ethane and $\mathrm{CO}_{2}$. The $\delta^{13} \mathrm{C}$ value of the methane is -67.5 (\%o, PDB), suggesting a biogenic or possibly a mixed biogenic-thermogenic source. This is remarkably similar to the $\delta^{13} \mathrm{C}$ value of methane reported by Suess and Whiticar (1989) from the Oregon Cascadia margin, suggesting a similar or common source. The $\delta^{18} \mathrm{O}$ value of the $\mathrm{H}_{2} \mathrm{O}$ which includes $\sim 6 \%$ admixed seawater is $+2.77\left(\%\right.$, SMOW); the $\delta^{18} \mathrm{O}$ value of the pure hydrate $\mathrm{H}_{2} \mathrm{O}$, assuming $0 \%$ o for the seawater, is thus $2.9 \%$. This value is similar to the experimentally determined oxygen isotope fractionation factor of 1.0026 in a gas hydrate-water system (Davidson et al., 1983) and basically the same as the experimentally determined oxygen isotope fractionation factor in the ice-water system (Craig and Hom, 1969; Suzuki and Kimura, 1973).

\section{REFERENCES}

Bottinga, Y., and Craig, H., 1969. Oxygen isotope fractionation between $\mathrm{CO}_{2}$ and water and the isotopic composition of marine atmospheric $\mathrm{CO}_{2}$. Earth Planet. Sci. Lett., 5:285-295.
Burke, W.H., Denison, R.E., Hetherington, E.A., Koepnick, R.B., Nelson, H.F., and Otto, J.B., 1982. Variation of seawater ${ }^{87} \mathrm{Sr} /{ }^{86} \mathrm{Sr}$ throughout Phanerozoic time. Geology, 10:516-519.

Craig, H., and Hom, B., 1969. Relationship between deuterium, oxygen-18 and chlorinity in the formation of sea ice. Trans. Am. Geophys. Union, 49:216-217.

Davidson, D.W., Leaist, D.G., and Hesse, R., 1983. Oxygen-18 enrichment in water of a clathrate hydrate. Geochim. Cosmochim. Acta., 47:22932295.

Elderfield, H., and Gieskes, J.M., 1982. Sr isotopes in interstitial waters of marine sediments from Deep Sea Drilling Project cores. Nature, 300: 493-497.

Gieskes, J.M., Gamo, T., and Brumsack, H., 1991. Chemical methods for interstitial water analysis aboard JOIDES Resolution. ODP Tech. Note, 15.

Suess, E., and Whiticar, M.J., 1989. Methane-derived $\mathrm{CO}_{2}$ in pore fluids expelled from the Oregon subduction zone. Palaeogeogr., Palaeoclimatol., Palaeoecol., 71:119-136.

Suzuki, T., and Kimura, T., 1973. D/H and ${ }^{18} \mathrm{O} / 16 \mathrm{O}$ fractionation in ice-water systems. Mass Spectros. (Tokyo), 21:229-233.

Date of initial receipt: 23 August 1994

Date of acceptance: 28 February 1995

Ms 146SR-211

Table 1. Chemical composition of pore fluid samples from Site 888.

\begin{tabular}{|c|c|c|c|c|c|c|c|}
\hline $\begin{array}{l}\text { Core, section, } \\
\text { interval }(\mathrm{cm})\end{array}$ & $\begin{array}{l}\text { Depth } \\
\text { (mbsf) }\end{array}$ & $\begin{array}{c}\mathrm{Cl} \\
(\mathrm{mM})\end{array}$ & $\begin{array}{l}\mathrm{Ca} / \mathrm{Cl} \\
\left(10^{-2}\right)\end{array}$ & $\begin{array}{c}\mathrm{Li} \\
(\mu \mathrm{M})\end{array}$ & $\begin{array}{l}\mathrm{Li} / \mathrm{Cl} \\
\left(10^{-4}\right)\end{array}$ & $\begin{array}{c}\mathrm{Sr} \\
(\mu \mathrm{M})\end{array}$ & $\begin{array}{l}\mathrm{Sr} / \mathrm{Cl} \\
\left(10^{-4}\right)\end{array}$ \\
\hline \multicolumn{8}{|l|}{ 146-888B- } \\
\hline IH-2, 143-150 & 2.9 & 545 & 1.88 & 23.7 & 0.43 & 91.0 & 1.67 \\
\hline $1 \mathrm{H}-3,105-110$ & 4.0 & 546 & 1.89 & 30.6 & 0.56 & & \\
\hline $2 \mathrm{H}-2,145-150$ & 8.5 & 552 & 2.08 & 11.3 & 0.20 & 101.6 & 1.84 \\
\hline $2 \mathrm{H}-3,53-58$ & 9.1 & 551 & 2.12 & 23.7 & 0.43 & 85.2 & 1.55 \\
\hline $3 \mathrm{H}-2,145-150$ & 18.0 & 562 & 2.16 & 23.7 & 0.42 & 81.1 & 1.44 \\
\hline $4 \mathrm{H}-4,145-150$ & 30.4 & 561 & 2.20 & 10.0 & 0.18 & 91.4 & 1.63 \\
\hline $5 \mathrm{H}-4,143-150$ & 39.9 & 564 & 2.27 & 16.8 & 0.30 & 98.4 & 1.74 \\
\hline $6 \mathrm{H}-5,140-150$ & 50.9 & 565 & 2.08 & 10.0 & 0.18 & 96.0 & 1.70 \\
\hline $7 \mathrm{H}-3,140-150$ & 57.4 & 568 & 1.84 & 13.4 & 0.24 & 92.1 & 1.62 \\
\hline $8 \mathrm{H}-4,140-150$ & 68.4 & 571 & 1.71 & 23.7 & 0.42 & 48.4 & 0.85 \\
\hline $9 \mathrm{H}-3,140-150$ & 76.4 & 559 & 1.65 & 22.5 & 0.40 & 48.4 & 0.87 \\
\hline $10 \mathrm{H}-4,140-150$ & 87.4 & 560 & 1.69 & 20.3 & 0.36 & 56.6 & 1.01 \\
\hline $11 \mathrm{H}-3,142-152$ & 95.3 & 559 & 1.50 & 10.0 & 0.18 & 93,4 & 1.67 \\
\hline $12 \mathrm{H}-2,139-149$ & 103.4 & 558 & 1.31 & 23.7 & 0.42 & 97.5 & 1.75 \\
\hline $14 \mathrm{H}-4,140-150$ & 124.0 & 556 & 1.84 & 19.9 & 0.36 & 105.5 & 1.90 \\
\hline $15 \mathrm{H}-4,138-150$ & 133.5 & 554 & 1.96 & 24.8 & 0.45 & 85.2 & 1.54 \\
\hline $16 \mathrm{H}-5,138-150$ & 144.5 & 554 & 1.73 & 16.8 & 0.30 & 91.4 & 1.65 \\
\hline $17 \mathrm{H}-4,140-150$ & 152.5 & 553 & 1.49 & 10.0 & 0.18 & 75.0 & 1.36 \\
\hline $18 X-2,135-150$ & 159.0 & 545 & 1.40 & 16.8 & 0.31 & 105.7 & 1.94 \\
\hline $19 X-5,1-15$ & 171.6 & 543 & 1.55 & 10.0 & 0.18 & 83.2 & 1.53 \\
\hline $24 \mathrm{H}-1,135-150$ & 214.3 & 552 & 1.23 & 16.8 & 0.30 & 101.6 & 1.84 \\
\hline $25 \mathrm{H}-3,135-150$ & 221.8 & 552 & 1.22 & 29.4 & 0.53 & 123.7 & 2.24 \\
\hline $26 \mathrm{H}-3,135-150$ & 231.3 & 550 & 1.17 & 20.3 & 0.37 & 112.2 & 2.04 \\
\hline $27 \mathrm{H}-5,140-150$ & 241.6 & 546 & 1.09 & 19.7 & 0.36 & 114.0 & 2.09 \\
\hline $30 \mathrm{H}-4,130-150$ & 266.6 & 547 & 1.05 & 19.9 & 0.36 & 109.1 & 1.99 \\
\hline $31 \mathrm{H}-5,130-150$ & 275.1 & 554 & 1.02 & 27.2 & 0.49 & 108.1 & 1.95 \\
\hline $34 \mathrm{H}-1,130-150$ & 293.7 & 548 & 1.19 & 23.7 & 0.43 & 107.8 & 1,97 \\
\hline $36 \mathrm{H}-2,70-80$ & 312.2 & 550 & 1.03 & 30.6 & 0.56 & 107.8 & 1.96 \\
\hline $40 \mathrm{H}-5,130-150$ & 355.3 & 552 & 1.17 & 30.6 & 0.55 & 103.5 & 1.88 \\
\hline $44 X-4,1-15$ & 389.5 & 556 & 1.44 & 30.6 & 0.55 & 128.3 & 2.31 \\
\hline $45 X-1,135-150$ & 396.4 & 559 & 1.44 & 32.0 & 0.57 & 122.1 & 2.18 \\
\hline $48 \mathrm{X}-\mathrm{CC}$ & 423.5 & 563 & 1.23 & 23.7 & 0.42 & 116.0 & 2.06 \\
\hline $53 \mathrm{X}-\mathrm{CC}, 3-18$ & 460.5 & 563 & 1.62 & 27.5 & 0.49 & 120.4 & 2.14 \\
\hline $54 X-1,130-150$ & 470.7 & 563 & 1.71 & 61.5 & 1.09 & 105.7 & 1.88 \\
\hline $57 X-1,130-150$ & 497.5 & 556 & 2.19 & 44.3 & 0.80 & 116.0 & 2.09 \\
\hline $58 X-3,1-20$ & 508.1 & 551 & 2.42 & & & 107.8 & 1.96 \\
\hline $59 \mathrm{P}-1,2-14$ & 514.0 & 545 & 2.47 & 51.5 & 0.94 & 113.9 & 2.09 \\
\hline $60 X-1,30-40$ & 515.3 & 563 & 2.39 & & & & \\
\hline $61 X-1,1-10$ & 522.8 & 556 & 2.24 & 55.0 & 0.99 & 105.7 & 1.90 \\
\hline $62 X-2,130-150$ & 534.5 & 564 & 2.66 & 46.4 & 0.82 & 121.5 & 2.15 \\
\hline $63 X-2,130-150$ & 543.2 & 562 & 2.90 & 37.9 & 0.67 & 120.1 & 2.14 \\
\hline $64 \mathrm{X}-1,130-150$ & 550.5 & 562 & 3.07 & & & 111.9 & 1.99 \\
\hline $65 X-5,1-25$ & 564.1 & 562 & 2.83 & 32.3 & 0.57 & 126.2 & 2.25 \\
\hline
\end{tabular}


Table 2. Chemical composition of pore fluid samples from Sites 889 and 890 .

\begin{tabular}{|c|c|c|c|c|c|c|c|}
\hline $\begin{array}{l}\text { Core, section, } \\
\text { interval }(\mathrm{cm})\end{array}$ & $\begin{array}{l}\text { Depth } \\
\text { (mbsf) }\end{array}$ & $\begin{array}{c}\mathrm{Cl} \\
(\mathrm{mM})\end{array}$ & $\begin{array}{l}\mathrm{Ca} / \mathrm{Cl} \\
\left(10^{-2}\right)\end{array}$ & $\begin{array}{c}\mathrm{Li} \\
(\mu \mathrm{M})\end{array}$ & $\begin{array}{l}\mathrm{Li} / \mathrm{Cl} \\
\left(10^{-4}\right)\end{array}$ & $\begin{array}{c}\mathrm{Sr} \\
(\mu \mathrm{M})\end{array}$ & $\begin{array}{l}\mathrm{Sr} / \mathrm{Cl} \\
\left(10^{-4}\right)\end{array}$ \\
\hline $\begin{array}{l}146-890 \mathrm{~B}- \\
1 \mathrm{H}-2,145-150 \\
1 \mathrm{H}-3,145-150 \\
2 \mathrm{H}-2,63-68 \\
2 \mathrm{H}-3,53-58 \\
2 \mathrm{H}-6,145-150 \\
3 \mathrm{H}-2,143-150 \\
3 \mathrm{H}-5,150-155 \\
5 \mathrm{H}-5,0-10\end{array}$ & $\begin{array}{r}3.0 \\
4.5 \\
9.4 \\
10.8 \\
16.5 \\
19.7 \\
24.2 \\
43.9\end{array}$ & $\begin{array}{l}544 \\
541 \\
532 \\
526 \\
522 \\
515 \\
508 \\
473\end{array}$ & $\begin{array}{l}1.27 \\
0.94 \\
0.62 \\
0.63 \\
0.42 \\
0.30 \\
0.37 \\
0.30\end{array}$ & $\begin{array}{l}26.2 \\
21.7 \\
28.6 \\
28.6 \\
27.9 \\
21.7 \\
24.4 \\
32.1\end{array}$ & $\begin{array}{l}0.48 \\
0.40 \\
0.54 \\
0.54 \\
0.53 \\
0.42 \\
0.48 \\
0.68\end{array}$ & $\begin{array}{l}76.1 \\
69.0 \\
64.3 \\
61.4 \\
58.0 \\
53.9 \\
52.3 \\
51.7\end{array}$ & $\begin{array}{l}1.40 \\
1.28 \\
1.21 \\
1.17 \\
1.11 \\
1.05 \\
1.03 \\
1.09\end{array}$ \\
\hline $\begin{array}{l}146-889 \mathrm{~A}- \\
1 \mathrm{H}-2,1-6 \\
1 \mathrm{H}-4,145-150 \\
2 \mathrm{H}-5,145-150 \\
3 \mathrm{H}-5,1-6 \\
4 \mathrm{H}-5,143-150 \\
5 \mathrm{H}-3,143-150 \\
6 \mathrm{H}-5,145-150 \\
7 \mathrm{H}-2,143-150 \\
8 \mathrm{H}-3,140-150 \\
9 \mathrm{H}-6,137-150 \\
10 \mathrm{H}-4,140-150 \\
11 \mathrm{H}-1,140-150 \\
11 \mathrm{H}-2,130-150 \\
12 \mathrm{H}-4,140-150 \\
13 \mathrm{H}-\mathrm{CC}, 0-5 \\
14 \mathrm{H}-1,153-158 \\
15 \mathrm{P}-1,6-13 \\
17 \mathrm{X}-2,70-80 \\
18 \mathrm{X}-4,36-46 \\
22 \mathrm{X}-7,0-20 \\
24 \mathrm{X}-5,120-140 \\
25 \mathrm{X}-2,0-20 \\
26 \mathrm{X}-4,130-150 \\
28 \mathrm{X}-4,80-100 \\
30 \mathrm{X}-4,130-150 \\
31 \mathrm{X}-5,140-160 \\
32 \mathrm{X}-1,130-150 \\
36 \mathrm{X}-1,99-114 \\
40 \mathrm{X}-4,45-65 \\
41 \mathrm{X}-3,130-150 \\
43 \mathrm{X}-2,0-5 \\
44 \mathrm{X}-1,0-15\end{array}$ & $\begin{array}{r}21.6 \\
26.0 \\
35.5 \\
45.0 \\
56.0 \\
62.5 \\
75.0 \\
80.0 \\
91.0 \\
103.4 \\
109.9 \\
115.0 \\
116.4 \\
125.0 \\
128.0 \\
128.5 \\
129.1 \\
132.4 \\
144.5 \\
179.8 \\
195.1 \\
198.8 \\
212.7 \\
222.6 \\
233.7 \\
244.0 \\
248.0 \\
267.7 \\
306.6 \\
316.4 \\
329.9 \\
337.3\end{array}$ & $\begin{array}{l}528 \\
521 \\
499 \\
488 \\
462 \\
456 \\
442 \\
432 \\
424 \\
412 \\
405 \\
401 \\
\\
398 \\
391 \\
378 \\
363 \\
379 \\
388 \\
378 \\
366 \\
364 \\
370 \\
372 \\
358 \\
352 \\
360 \\
364 \\
365 \\
362 \\
367 \\
376\end{array}$ & $\begin{array}{l}0.66 \\
0.57 \\
0.61 \\
0.59 \\
0.44 \\
0.27 \\
0.50 \\
0.61 \\
0.73 \\
0.88 \\
0.95 \\
0.99 \\
0.99 \\
1.04 \\
1.07 \\
1.21 \\
0.96 \\
0.87 \\
1.06 \\
1.19 \\
1.07 \\
1.13 \\
1.11 \\
1.13 \\
1.07 \\
1.14 \\
1.17 \\
1.20 \\
1.39 \\
1.56 \\
1.49\end{array}$ & $\begin{array}{l}19.8 \\
22.7 \\
22.7 \\
22.7 \\
22.7 \\
22.7 \\
26.6 \\
31.5 \\
31.5 \\
31.5 \\
35.8 \\
40.2 \\
40.2 \\
41.0 \\
44.6 \\
50.0\end{array}$ & $\begin{array}{l}0.38 \\
0.44 \\
0.45 \\
0.47 \\
0.49 \\
0.50 \\
0.60 \\
0.73 \\
0.74 \\
0.76 \\
0.88 \\
1.00 \\
\\
1.03 \\
1.14 \\
1.32\end{array}$ & $\begin{array}{l}74.7 \\
71.8 \\
66.5 \\
59.8 \\
52.0 \\
52.0 \\
54.4 \\
56.7 \\
56.7 \\
54.4 \\
52.0 \\
52.0 \\
38.4 \\
52.0 \\
52.0 \\
50.1 \\
38.1 \\
50.4 \\
46.3 \\
61.5 \\
52.7 \\
59.2 \\
56.7 \\
49.7 \\
53.2 \\
56.1 \\
58.7 \\
60.5 \\
60.8 \\
62.6\end{array}$ & $\begin{array}{l}1.41 \\
1.38 \\
1.33 \\
1.23 \\
1.13 \\
1.14 \\
1.23 \\
1.31 \\
1.34 \\
1.32 \\
1.28 \\
1.30 \\
1.31 \\
1.33 \\
1.33 \\
1.05 \\
1.30 \\
1.22 \\
1.68 \\
1.45 \\
1.60 \\
1.52 \\
1.39 \\
1.51 \\
1.56 \\
1.61 \\
1.66 \\
1.68 \\
1.71\end{array}$ \\
\hline $\begin{array}{l}\text { 146-889B- } \\
\text { 3R-1, 79-94 } \\
4 \mathrm{R}-2,45-60 \\
\text { 5R-1, 26-36 } \\
6 \mathrm{R}-1,54-69 \\
7 \mathrm{R}-1,103-126 \\
8 \mathrm{R}-2,130-150 \\
\text { 9R-1, 130-150 } \\
10 \mathrm{R}-2,0-20 \\
\text { 12R-1, 130-150 } \\
\text { 13R-2, 35-55 } \\
\text { 14R-1, 111-136 } \\
\text { 15R-1, 125-150 } \\
\text { 17R-1, 0-15 } \\
\text { 18R-1, 0-10 } \\
\text { 20R-CC, } 0-3\end{array}$ & $\begin{array}{l}216.5 \\
227.3 \\
235.1 \\
244.9 \\
255.0 \\
265.6 \\
274.3 \\
282.0 \\
299.6 \\
309.1 \\
317.0 \\
326.0 \\
342.2 \\
351.1 \\
377.6\end{array}$ & $\begin{array}{l}368 \\
380 \\
370 \\
365 \\
362 \\
372 \\
370 \\
371 \\
364 \\
365 \\
375 \\
368 \\
379 \\
369 \\
366\end{array}$ & $\begin{array}{l}0.98 \\
1.17 \\
1.20 \\
1.17 \\
1.16 \\
1.15 \\
1.12 \\
1.29 \\
1.21 \\
1.30 \\
1.35 \\
1.50 \\
1.56 \\
1.41 \\
1.74\end{array}$ & $\begin{array}{c}79.9 \\
84.0 \\
84.7 \\
90.6 \\
84.0 \\
99.2 \\
110.3 \\
105.9 \\
127.8 \\
106.0 \\
195.2\end{array}$ & $\begin{array}{l}2.10 \\
2.27 \\
2.32 \\
2.50 \\
2.26 \\
2.68 \\
\\
3.03 \\
2.90 \\
3.47 \\
\\
2.87 \\
5.33\end{array}$ & $\begin{array}{l}63.3 \\
52.5 \\
58.7 \\
59.8 \\
54.5 \\
\\
56.6 \\
59.1 \\
\\
66.8 \\
62.7 \\
70.9 \\
60.7 \\
77.6\end{array}$ & $\begin{array}{l}1.67 \\
1.42 \\
1.61 \\
1.65 \\
1.47 \\
\\
1.53 \\
1.62 \\
\\
1.78 \\
1.70 \\
1.87 \\
1.64 \\
2.12\end{array}$ \\
\hline
\end{tabular}


Table 3. Chemical composition of pore fluid samples from Site 891 .

\begin{tabular}{|c|c|c|c|c|c|c|c|}
\hline $\begin{array}{l}\text { Core, section, } \\
\text { interval }(\mathrm{cm})\end{array}$ & $\begin{array}{l}\text { Depth } \\
\text { (mbsf) }\end{array}$ & $\underset{(\mathrm{mM})}{\mathrm{Cl}}$ & $\begin{array}{l}\mathrm{Ca} / \mathrm{Cl} \\
\left(10^{-2}\right)\end{array}$ & $\underset{(\mu \mathrm{M})}{\mathrm{Li}}$ & $\begin{array}{l}\mathrm{Li} / \mathrm{Cl} \\
\left(10^{-4}\right)\end{array}$ & $\begin{array}{c}\mathrm{Sr} \\
(\mu \mathrm{M})\end{array}$ & $\begin{array}{l}\mathrm{Sr} / \mathrm{Cl} \\
\left(10^{-4}\right)\end{array}$ \\
\hline \multicolumn{8}{|l|}{ 146-891A- } \\
\hline $1 \mathrm{H}-1,140-150$ & 2.9 & 545 & 1.05 & 26.2 & 0.48 & 93.4 & 1.71 \\
\hline $1 \mathrm{H}-3,103-113$ & 4.0 & 527 & 1.17 & 21.7 & 0.41 & 95.5 & 1.81 \\
\hline $2 \mathrm{H}-1,109-119$ & 5.8 & 546 & 0.92 & 21.7 & 0.40 & 87.2 & 1.60 \\
\hline $3 \mathrm{H}-2,53-68$ & 9.3 & 551 & 1.01 & 21.7 & 0.39 & 108.7 & 1.97 \\
\hline \multicolumn{8}{|l|}{ 146-891B- } \\
\hline $3 \mathrm{X}-\mathrm{CC}$ & 20.8 & 532 & 1.72 & 38.4 & 0.72 & 89.3 & $\begin{array}{l}1.68 \\
1.75\end{array}$ \\
\hline $4 X-C C, 0-5$ & 29.6 & 533 & 1.69 & & & 93.4 & 1.75 \\
\hline $8 X-1,15-20$ & 65.4 & 537 & 1.81 & 23.1 & 0.43 & 93.4 & 1.74 \\
\hline $11 X-2,16-21$ & 92.3 & 540 & 1.85 & 26.6 & 0.49 & 89.3 & 1.65 \\
\hline $14 X-1,54-64$ & 110.0 & 540 & 1.83 & 23.2 & 0.43 & 95.5 & 1.77 \\
\hline $15 X-1,82-90$ & 119.2 & 545 & 1.72 & 34.2 & 0.63 & 90.7 & 1.66 \\
\hline $16 X-1,57-67$ & 127.9 & 545 & 1.60 & 30.6 & 0.56 & 97.5 & 1.79 \\
\hline $18 \mathrm{X}-\mathrm{CC}, 26-37$ & 148.4 & 546 & 1.80 & 18.9 & 0.35 & 105.7 & 1.94 \\
\hline $19 \mathrm{X}-1,0-10$ & 153.9 & 541 & 1.80 & 21.7 & 0.40 & 102.3 & 1.89 \\
\hline $20 \mathrm{X}-1,28-38$ & 163.0 & 545 & 1.81 & 15.9 & 0.29 & 112.5 & 2.06 \\
\hline $21 \mathrm{~N}-\mathrm{CC}, 0-5$ & 172.1 & 546 & 1.74 & & & 105.7 & 1.94 \\
\hline $22 X-1,55-67$ & 176.7 & 546 & 1.62 & 21.7 & 0.40 & 97.5 & 1.79 \\
\hline $23 X-1,50-60$ & 181.1 & 545 & 1.54 & & & & \\
\hline $25 \mathrm{X}-\mathrm{CC}, 13-20$ & 198.9 & 555 & 1.01 & 10.0 & 0.18 & 89.3 & 1.61 \\
\hline $26 \mathrm{X}-1,42-46$ & 207.5 & 554 & & & & 97.5 & 1.76 \\
\hline $27 X-1,15-25$ & 216.1 & 561 & 0.90 & 27.3 & 0.49 & 107.4 & 1.91 \\
\hline $28 \mathrm{X}-1,32-50$ & 225.0 & 557 & 0.92 & 14.3 & 0.26 & 101.6 & 1.82 \\
\hline $29 X-1,22-29$ & 233.8 & 556 & 0.96 & 3.1 & 0.06 & 103.7 & 1.86 \\
\hline $30 \times-1,75-83$ & 238.4 & 557 & 0.99 & 18.2 & 0.33 & 101.6 & 1.83 \\
\hline $31 X-1,135-150$ & 243.8 & 562 & 0.97 & 21.7 & 0.39 & 95.5 & 1.70 \\
\hline $32 X-1,11-17$ & 251.5 & 561 & 0.99 & 25.1 & 0.45 & & \\
\hline $33 X-1,0-17$ & 260.2 & 561 & 1.14 & 36.5 & 0.65 & 108.6 & 1.94 \\
\hline $34 X-1,100-115$ & 264.1 & 563 & 0.90 & 10.0 & $0.18^{\circ}$ & & \\
\hline $35 X-1,16-28$ & 269.2 & 558 & 0.91 & & & 99.6 & 1.79 \\
\hline $36 \mathrm{P}-1,0-6$ & 277.8 & 557 & 1.02 & 28.6 & 0.51 & & \\
\hline $38 X-2,0-18$ & 287.9 & 555 & 1.03 & 40.7 & 0.73 & 108.6 & 1.96 \\
\hline $39 \mathrm{X}-1,101-118$ & 296.1 & 552 & 1.01 & 50.4 & 0.91 & 103.7 & 1.88 \\
\hline $40 X-1,0-11$ & 304.0 & 557 & 1.02 & 39.1 & 0.70 & 103.7 & 1.86 \\
\hline $41 X-1,110-135$ & 314.1 & 556 & 1.32 & 53.8 & 0.97 & 110.3 & 1.98 \\
\hline $41 X-1135-150$ & 314.2 & 553 & 1.47 & 52.1 & 0.94 & 116.0 & 2.10 \\
\hline $42 X-1,0-18$ & 321.6 & 560 & 2.04 & 19.6 & 0.35 & 103.6 & 1.85 \\
\hline $43 X-1,10-27$ & 330.5 & 562 & 1.12 & 18.2 & 0.32 & 109.8 & 1.95 \\
\hline $45 X-1.6-14$ & 348.3 & 554 & & 18.2 & 0.33 & & \\
\hline $47 X-1.95-110$ & 367.1 & 559 & 1.20 & 48.1 & 0.86 & 137.5 & 2.46 \\
\hline $48 X-1,0-15$ & 375.0 & 561 & 1.09 & & & 126.2 & 2.25 \\
\hline $49 \times-1,23-29$ & 384.1 & 550 & & & & 114.2 & 2.08 \\
\hline $50 X-1,17-20$ & 393.0 & 555 & & & & 128.3 & 2.31 \\
\hline $52 X-1,7-21$ & 410.6 & 553 & 1.68 & 31.5 & 0.57 & 140.9 & 2.55 \\
\hline $55 X-2,130-150$ & 439.0 & 546 & 1.72 & 50.2 & 0.92 & 123.7 & 2.27 \\
\hline $56 \mathrm{X}-1,72-89$ & 446.5 & 546 & 1.06 & 44.5 & 0.81 & 116.0 & 2.12 \\
\hline $58 X-2,15-27$ & 464.6 & 557 & 1.10 & 34.5 & 0.62 & 116.4 & 2.09 \\
\hline
\end{tabular}

Table 4. Chemical composition of pore fluid samples from Hole 892A.

\begin{tabular}{|c|c|c|c|c|c|c|c|}
\hline $\begin{array}{l}\text { Core, section, } \\
\text { interval }(\mathrm{cm})\end{array}$ & $\begin{array}{l}\text { Depth } \\
\text { (mbsf) }\end{array}$ & $\underset{(\mathrm{mM})}{\mathrm{Cl}}$ & $\begin{array}{l}\mathrm{Ca} / \mathrm{Cl} \\
\left(10^{-2}\right)\end{array}$ & $\begin{array}{c}\mathrm{Li} \\
(\mu \mathrm{M})\end{array}$ & $\begin{array}{l}\mathrm{Li} / \mathrm{Cl} \\
\left(10^{-4}\right)\end{array}$ & $\begin{array}{c}\mathrm{Sr} \\
(\mu \mathrm{M})\end{array}$ & $\begin{array}{l}\mathrm{Sr} / \mathrm{Cl} \\
\left(10^{-4}\right)\end{array}$ \\
\hline \multicolumn{8}{|l|}{$146-892 \mathrm{~A}-$} \\
\hline $1 X-5,10-13$ & 6.1 & 483 & 0.70 & 28.4 & 0.59 & 70.7 & 1.46 \\
\hline $2 X-3,0-11$ & 12.6 & 468 & 0.90 & 28.7 & 0.61 & 75.4 & 1.61 \\
\hline $3 X-2,0-10$ & 20.6 & 557 & 1.00 & 66.7 & 1.20 & 164.3 & 2.95 \\
\hline $3 X-3,0-10$ & 22.1 & 552 & 0.80 & 71.6 & 1.30 & 159.6 & 2.89 \\
\hline $4 X-2,0-10$ & 30.1 & 523 & 1.00 & 84.3 & 1.61 & 169.0 & 3.23 \\
\hline $6 X-2,0-10$ & 40.6 & 544 & 1.00 & 110.3 & 2.03 & 192.4 & 3.54 \\
\hline $6 \mathrm{X}-2,10-30$ & 40.7 & 545 & 1.10 & 116.4 & 2.14 & 199.4 & 3.66 \\
\hline $7 X-5,140-150$ & 55.9 & 507 & 1.20 & 143.4 & 2.83 & 208.7 & 4.12 \\
\hline $8 X-3,0-10$ & 61.0 & 534 & 1.20 & 155.8 & 2.92 & 229.8 & 4.30 \\
\hline $9 X-1,69-74$ & 68.2 & 480 & 1.30 & & & 232.7 & 4.85 \\
\hline $11 X-2,0-10$ & 79.6 & 517 & 1.30 & 176.3 & 3.41 & 267.2 & 5.17 \\
\hline $11 \mathrm{X}-2,70-95$ & 80.3 & 516 & 1.40 & 186.8 & 3.62 & 262.5 & 5.09 \\
\hline $12 \times-1,0-7$ & 87.5 & 489 & 1.50 & & & 245.0 & 5.02 \\
\hline $13 X-4,0-25$ & 101.6 & 504 & 1.40 & 188.4 & 3.74 & 259.4 & 5.15 \\
\hline $13 X-6,69-82$ & 105.3 & 501 & 1.40 & 161.6 & 3.23 & 213.2 & 4.26 \\
\hline $14 X-1,35-38$ & 106.9 & 491 & 1.60 & 215.3 & 4.38 & 234.8 & 4.78 \\
\hline $15 X-1,88-100$ & 116.9 & 497 & 1.40 & 168.5 & 3.39 & 223.7 & 4.50 \\
\hline $16 X-1,34-43$ & 125.8 & 493 & 1.40 & 168.3 & 3.41 & 260.2 & 5.28 \\
\hline $17 X-2,3-13$ & 136.6 & 481 & 1.40 & 156.3 & 3.25 & 207.5 & 4.31 \\
\hline $18 X-1,115-140$ & 145.8 & 487 & 1.40 & 167.9 & 3.45 & 264.9 & 5.44 \\
\hline $18 X-1,140-150$ & 146.0 & 487 & 1.40 & 161.0 & 3.31 & 260.2 & 5.34 \\
\hline $20 \mathrm{X}-2,0-10$ & 165.1 & 489 & 1.40 & 156.9 & 3.21 & 278.9 & 5.70 \\
\hline $20 \times-3,0-10$ & 166.5 & 496 & 1.40 & 154.0 & 3.11 & 261.4 & 5.27 \\
\hline
\end{tabular}


Table 5. Chemical composition of pore fluid samples from Hole 892D.

\begin{tabular}{|c|c|c|c|c|c|c|c|c|c|c|c|}
\hline $\begin{array}{l}\text { Core, section, } \\
\text { interval }(\mathrm{cm})\end{array}$ & $\begin{array}{l}\text { Depth } \\
\text { (mbsf) }\end{array}$ & $\underset{(\mathrm{mM})}{\mathrm{Cl}}$ & $\underset{(\mathrm{mM})}{\mathrm{Ca}}$ & $\begin{array}{l}\mathrm{Ca} / \mathrm{Cl} \\
\left(10^{-2}\right)\end{array}$ & $\underset{(\mathrm{mM})}{\mathrm{Mg}}$ & $\underset{(\mathrm{mM})}{\mathrm{K}}$ & $\underset{(\mathrm{mM})}{\mathrm{Na}}$ & $\begin{array}{c}\mathrm{Li} \\
(\mu \mathrm{M})\end{array}$ & $\begin{array}{l}\mathrm{Li} / \mathrm{Cl} \\
\left(10^{-4}\right)\end{array}$ & $\begin{array}{c}\mathrm{Sr} \\
(\mu \mathrm{M})\end{array}$ & $\begin{array}{l}\mathrm{Sr} / \mathrm{Cl} \\
\left(10^{-4}\right)\end{array}$ \\
\hline \multicolumn{12}{|l|}{ 146-892D- } \\
\hline $1 X-1,9-12$ & 0.1 & 547 & 9.68 & 1.80 & 49.42 & 13.10 & 474 & 33.3 & 0.61 & 87.1 & 1.59 \\
\hline $2 X-1,145-150$ & 10.0 & 539 & 3.37 & 0.60 & 39.93 & 12.62 & 461 & 31.2 & 0.58 & 80.1 & 1.49 \\
\hline $2 X-2,140-150$ & 11.4 & 447 & 2.90 & 0.70 & 30.75 & 10.62 & 388 & 27.8 & 0.62 & 63.7 & 1.43 \\
\hline $3 X-1,40-45$ & 18.4 & 433 & 3.26 & 0.80 & 31.81 & 10.70 & 376 & 28.8 & 0.67 & 54.2 & 1.25 \\
\hline $4 X-2,4-11$ & 29.0 & 575 & & & & 10.87 & & 82.0 & 1.43 & 161.7 & 2.81 \\
\hline $4 X-2,21-30$ & 29.2 & 505 & 5.07 & 1.00 & 32.93 & 10.01 & 441 & 61.0 & 1.21 & 131.3 & 2.60 \\
\hline $4 X-3,80-88$ & 30.8 & 570 & & & & 10.85 & & 84.1 & 1.48 & 180.7 & 3.17 \\
\hline $5 X-2,140-150$ & 39.9 & 560 & 5.56 & 1.00 & 33.97 & 10.23 & 495 & 104.3 & 1.86 & 197.0 & 3.52 \\
\hline $6 \mathrm{X}-4,140-150$ & 52.4 & 550 & 5.91 & 1.10 & 32.52 & 9.70 & 479 & 126.7 & 2.30 & 211.1 & 3.84 \\
\hline $7 X-3,142-150$ & 58.2 & 551 & 3.66 & 0.70 & 31.92 & 9.40 & 486 & 147.2 & 2.67 & 234.5 & 4.26 \\
\hline $8 X-3,0-10$ & 64.7 & 541 & 4.10 & 0.80 & 28.97 & 9.05 & 481 & 154.7 & 2.86 & 239.1 & 4.42 \\
\hline $9 \times-4,0-15$ & 73.8 & 500 & 4.64 & 0.90 & 23.71 & 7.66 & 448 & 163.0 & 3.26 & 250.8 & 5.02 \\
\hline $10 \times-4,0-15$ & 104.5 & 496 & & & & 5.90 & & 175.2 & 3.53 & 273.7 & 5.52 \\
\hline $11 X-1,130-150$ & 110.9 & 505 & 4.67 & 0.90 & 21.31 & 6.67 & 459 & 170.7 & 3.38 & 277.0 & 5.49 \\
\hline $12 X-3,130-150$ & 123.4 & 488 & 4.20 & 0.90 & 21.48 & 6.55 & 445 & 165.2 & 3.39 & 260.2 & 5.33 \\
\hline $13 \mathrm{X}-\mathrm{CC}, 22-24$ & 128.5 & 484 & & & & & & & & 272.9 & 5.64 \\
\hline $14 X-1,115-130$ & 139.2 & 490 & 3.94 & 0.80 & 21.59 & 6.53 & 447 & 156.8 & 3.20 & 267.2 & 5.45 \\
\hline $15 X-2,110-135$ & 148.6 & 489 & 4.81 & 1.00 & 21.85 & 6.88 & & 160.5 & 3.28 & 246.2 & 5.03 \\
\hline $15 X-2,135-150$ & 148.9 & 492 & 4.00 & 0.80 & 21.45 & 6.50 & 451 & 147.7 & 3.00 & 267.2 & 5.43 \\
\hline $16 X-3,135-150$ & 161.4 & 484 & 3.56 & 0.70 & 21.96 & 6.47 & 442 & 141.0 & 2.91 & 267.2 & 5.52 \\
\hline $16 \times-5,135-150$ & 164.4 & 485 & 3.78 & 0.80 & 21.43 & 6.36 & 444 & 136.4 & 2.81 & 267.2 & 5.51 \\
\hline
\end{tabular}

Table 6. Strontium concentration and strontium and oxygen isotope ratios of pore fluids, Site 888.

\begin{tabular}{lrrrrr}
\hline $\begin{array}{c}\text { Core, section, } \\
\text { interval }(\mathrm{cm})\end{array}$ & $\begin{array}{r}\text { Depth } \\
(\mathrm{mbsf})\end{array}$ & $\begin{array}{c}\mathrm{Sr} \\
(\mu \mathrm{M})\end{array}$ & ${ }^{87} \mathrm{Sr}{ }^{86} \mathrm{Sr}$ & $2 \sigma$ & $\begin{array}{c}\delta^{18} \mathrm{O} \\
(\% \mathrm{SMOW})\end{array}$ \\
\hline 146-888B- & & & & & \\
$2 \mathrm{H}-2,140-150$ & 8.5 & 101.6 & 0.708904 & 18 & 0.04 \\
$5 \mathrm{H}-4,143-150$ & 39.9 & 98.4 & 0.708364 & 18 & 0.30 \\
$8 \mathrm{H}-4,140-150$ & 68.4 & 48.4 & 0.708524 & 21 & 0.20 \\
$12 \mathrm{H}-2,140-150$ & 103.4 & 97.5 & 0.708340 & 18 & -0.19 \\
$15 \mathrm{H}-4,138-150$ & 133.5 & 85.2 & 0.707825 & 23 & -0.51 \\
$19 \mathrm{X}-5,0-15$ & 171.6 & 83.2 & 0.707831 & 17 & -0.51 \\
$25 \mathrm{H}-3,135-150$ & 221.8 & 123.7 & 0.707779 & 16 & -0.65 \\
$34 \mathrm{H}-1,130-150$ & 293.7 & 107.8 & 0.708059 & 15 & -0.62 \\
$45 \mathrm{X}-1,135-150$ & 396.4 & 122.1 & 0.708380 & 23 & -0.79 \\
$53 \mathrm{X}-1,3-18$ & 460.5 & 120.4 & 0.708159 & 25 & -0.88 \\
$59 \mathrm{P}-1,2-14$ & 514.0 & 113.9 & 0.707813 & 16 & -1.23 \\
$61 \mathrm{X}-1,0-10$ & 522.8 & 105.7 & 0.707941 & 17 & -1.04 \\
$64 \mathrm{X}-1,130-150$ & 550.5 & 111.9 & 0.707590 & 25 & -1.07 \\
\hline
\end{tabular}

Table 7. Strontium concentration and strontium and oxygen isotope ratios of pore fluids, Sites 889 and 890 .

\begin{tabular}{|c|c|c|c|c|c|}
\hline $\begin{array}{l}\text { Core, section, } \\
\text { interval }(\mathrm{cm})\end{array}$ & $\begin{array}{l}\text { Depth } \\
\text { (mbsf) }\end{array}$ & $\begin{array}{c}\mathrm{Sr} \\
(\mu \mathrm{M})\end{array}$ & ${ }^{87} \mathrm{Sr} /{ }^{86} \mathrm{Sr}$ & $2 \sigma$ & $\begin{array}{c}\delta^{18} \mathrm{O} \\
(\% \mathrm{SMOW})\end{array}$ \\
\hline \multicolumn{6}{|l|}{ 146-890B- } \\
\hline $2 \mathrm{H}-2,63-68$ & 9.4 & 64.3 & 0.709002 & 17 & -0.22 \\
\hline $3 \mathrm{H}-5,150-155$ & 24.2 & 52.3 & 0.709018 & 16 & -0.34 \\
\hline $5 \mathrm{H}-5,0-10$ & 43.9 & 51.7 & 0.708887 & 19 & \\
\hline \multicolumn{6}{|l|}{$146-$} \\
\hline $889 \mathrm{~A}-3 \mathrm{H}-5,1-6$ & 45.0 & 59.8 & 0.708977 & 19 & \\
\hline $889 \mathrm{~A}-4 \mathrm{H}-5,143-150$ & 56.0 & 52.0 & 0.708899 & 16 & -0.63 \\
\hline $889 \mathrm{~A}-5 \mathrm{H}-3,143-150$ & 62.5 & 52.0 & 0.708826 & 18 & -0.77 \\
\hline $889 \mathrm{~A}-6 \mathrm{H}-5,145-150$ & 75.0 & 54.4 & & & -1.07 \\
\hline $889 \mathrm{~A}-8 \mathrm{H}-3,140-150$ & 91.0 & 56.7 & 0.708637 & 20 & -1.19 \\
\hline $889 \mathrm{~A}-11 \mathrm{H}-\mathrm{I}, 140-150$ & 115.0 & 52.0 & 0.708521 & 18 & -1.37 \\
\hline $889 \mathrm{~A}-12 \mathrm{H}-4,140-150$ & 125.0 & 52.0 & 0.708544 & 18 & \\
\hline $889 \mathrm{~A}-13 \mathrm{H}-\mathrm{CC}, 0-5$ & 128.0 & 52.0 & & & -1.49 \\
\hline $889 \mathrm{~A}-15 \mathrm{P}-1,6-13$ & 129.1 & 38.1 & 0.708622 & 17 & \\
\hline $889 \mathrm{~A}-17 \mathrm{X}-2,70-80$ & 132.4 & & 0.708514 & 15 & \\
\hline $889 \mathrm{~A}-18 \mathrm{X}-4,36-46$ & 144.5 & 50.4 & 0.708497 & 17 & -1.44 \\
\hline $889 \mathrm{~A}-24 \mathrm{X}-5,120-140$ & 195.1 & 61.5 & 0.708507 & 16 & -1.32 \\
\hline $889 \mathrm{~A}-26 \mathrm{X}-4,130-150$ & 212.7 & 59.2 & & & -1.44 \\
\hline $889 A-28 X-4,80-100$ & 222.6 & 56.7 & 0.708492 & 16 & -1.30 \\
\hline $889 \mathrm{~B}-4 \mathrm{R}-2,45-60$ & 227.3 & 63.3 & 0.708554 & 18 & -1.38 \\
\hline $889 \mathrm{~A}-30 \mathrm{X}-4,130-150$ & 233.7 & 49.7 & 0.708438 & 18 & \\
\hline $889 \mathrm{~A}-32 \mathrm{X}-1,130-150$ & 248.0 & 56.1 & 0.708402 & 16 & \\
\hline $889 \mathrm{~B}-8 \mathrm{R}-2,130-150$ & 265.6 & 54.5 & 0.708477 & 19 & \\
\hline $889 \mathrm{~A}-36 \mathrm{X}-1,99-114$ & 267.7 & 58.7 & & & -1.42 \\
\hline $889 \mathrm{~B}-9 \mathrm{R}-1,130-150$ & 274.3 & & 0.708449 & 15 & \\
\hline $889 A-40 X-4,45-65$ & 306.6 & 60.5 & 0.708237 & 18 & -1.43 \\
\hline $889 \mathrm{~A}-43 \mathrm{X}-2,0-5$ & 329.9 & 62.6 & 0.708224 & 16 & -1.40 \\
\hline $889 \mathrm{~B}-18 \mathrm{R}-1,0-10$ & 351.1 & 60.7 & 0.708213 & 18 & -1.53 \\
\hline $889 \mathrm{~B}-20 \mathrm{R}-\mathrm{CC}, 0-3$ & 377.6 & 77.6 & 0.707766 & 19 & -1.38 \\
\hline
\end{tabular}


Table 8. Strontium concentration and strontium and oxygen isotope ratios of pore fluids, Site 891 .

\begin{tabular}{|c|c|c|c|c|c|}
\hline $\begin{array}{l}\text { Core, section, } \\
\text { interval }(\mathrm{cm})\end{array}$ & $\begin{array}{l}\text { Depth } \\
\text { (mbsf) }\end{array}$ & $\begin{array}{c}\mathrm{Sr} \\
(\mu \mathrm{M})\end{array}$ & ${ }^{87} \mathrm{Sr} /{ }^{86} \mathrm{Sr}$ & $2 \sigma$ & $\begin{array}{c}\delta^{18} \mathrm{O} \\
(\% \text { SMOW) }\end{array}$ \\
\hline $\begin{array}{l}146-891 \mathrm{~A}- \\
2 \mathrm{H}-1,109-119\end{array}$ & 5.8 & 87.2 & 0.708561 & 18 & -0.12 \\
\hline $\begin{array}{l}\text { 146-891B- } \\
4 X-C C, 0-5 \\
14 X-1,54-64 \\
20 X-1,28-38 \\
23 X-1,50-60 \\
25 X-C C, 13-20 \\
26 X-1,42-46 \\
27 X-1,15-25 \\
29 X-1,22-29 \\
31 X-1,135-150 \\
33 X-1,0-17 \\
34 X-1,100-115 \\
35 X-1,16-28 \\
38 X-2,0-18 \\
42 X-1,0-18 \\
47 X-1,95-110 \\
50 X-1,17-20 \\
55 X-2,130-150 \\
56 X-1,72-89 \\
58 X-2,15-27\end{array}$ & $\begin{array}{l}29.6 \\
110.0 \\
163.0 \\
181.1 \\
198.9 \\
207.5 \\
216.1 \\
233.8 \\
243.8 \\
260.2 \\
264.1 \\
269.2 \\
287.9 \\
321.6 \\
367.1 \\
393.0 \\
439.0 \\
446.5 \\
464.6\end{array}$ & $\begin{array}{r}93.4 \\
95.5 \\
112.5 \\
\\
89.3 \\
97.5 \\
107.4 \\
103.7 \\
95.5 \\
108.6 \\
\\
99.6 \\
108.6 \\
103.6 \\
137.5 \\
128.3 \\
123.7 \\
116.0 \\
116.4\end{array}$ & $\begin{array}{l}0.709076 \\
0.708993 \\
0.708144 \\
0.708079 \\
0.708099 \\
\\
0.708199 \\
0.708270 \\
0.708308 \\
0.708325 \\
0.708349 \\
0.708135 \\
0.708037 \\
0.708300 \\
0.708207 \\
0.708316 \\
0.708153\end{array}$ & $\begin{array}{l}18 \\
17 \\
\\
25 \\
35 \\
37 \\
\\
\\
41 \\
18 \\
17 \\
20 \\
17 \\
\\
\\
18 \\
14 \\
21 \\
31 \\
20 \\
23\end{array}$ & $\begin{array}{l}-0.07 \\
-0.24 \\
-0.09 \\
-0.08 \\
-0.16 \\
-0.25 \\
-0.56 \\
-0.58 \\
-0.50 \\
-0.69 \\
-0.52 \\
-0.69 \\
-0.73 \\
-0.51 \\
-0.77 \\
-1.17 \\
-1.28 \\
-0.69\end{array}$ \\
\hline
\end{tabular}

Table 9. Strontium concentration and strontium and oxygen isotope ratios of pore fluids, Site 892.

\begin{tabular}{|c|c|c|c|c|c|}
\hline $\begin{array}{l}\text { Core, section, } \\
\text { interval }(\mathrm{cm})\end{array}$ & $\begin{array}{l}\text { Depth } \\
\text { (mbsf) }\end{array}$ & $\begin{array}{c}\mathrm{Sr} \\
(\mu \mathrm{M})\end{array}$ & ${ }^{87} \mathrm{Sr} /{ }^{86} \mathrm{Sr}$ & $2 \sigma$ & $\begin{array}{c}\delta^{18} \mathrm{O} \\
\text { (\% SMOW }\end{array}$ \\
\hline \multicolumn{6}{|l|}{ 146- } \\
\hline $892 D-1 X-1,9-12$ & 0.1 & 87.1 & & & -0.07 \\
\hline $892 \mathrm{D}-1 \mathrm{X}-1,145-150$ & 10.0 & 80.1 & 0.708878 & 19 & -0.17 \\
\hline $892 \mathrm{D}-2 \mathrm{X}-2,140-150$ & 11.4 & 63.7 & 0.708560 & 18 & 0.33 \\
\hline $892 \mathrm{~A}-2 \mathrm{X}-3,0-11$ & 12.6 & 75.4 & 0.708411 & 20 & -0.04 \\
\hline $892 \mathrm{~A}-3 \mathrm{X}-2,0-10$ & 20.6 & 164.3 & 0.707718 & 18 & -0.11 \\
\hline $892 \mathrm{~A}-4 \mathrm{X}-2,0-10$ & 30.1 & 169.0 & 0.707589 & 18 & -0.11 \\
\hline $892 \mathrm{~A}-6 \mathrm{X}-2,0-10$ & 40.6 & 192.4 & 0.707461 & 16 & -0.26 \\
\hline $892 \mathrm{D}-6 \mathrm{X}-4,140-150$ & 52.4 & 211.1 & 0.707461 & 20 & \\
\hline $892 \mathrm{~A}-7 \mathrm{X}-5,140-150$ & 55.9 & 208.7 & 0.707277 & 18 & -0.30 \\
\hline $892 \mathrm{~A}-8 \mathrm{X}-3,0-10$ & 61.0 & 229.8 & 0.707289 & 21 & -0.33 \\
\hline $892 D-8 X-3,0-10$ & 64.7 & 239.1 & 0.707268 & 18 & -0.49 \\
\hline $892 \mathrm{~A}-9 \mathrm{X}-1,69-74$ & 68.2 & 232.7 & 0.707168 & 18 & -0.32 \\
\hline $892 \mathrm{D}-9 \mathrm{X}-4,0-15$ & 73.8 & 250.8 & 0.707137 & 17 & -0.31 \\
\hline $892 \mathrm{~A}-11 \mathrm{X}-2,0-10$ & 79.6 & 267.2 & 0.707116 & 20 & -0.48 \\
\hline $892 \mathrm{~A}-13 \mathrm{X}-4,0-25$ & 101.6 & 259.4 & 0.707095 & 18 & -0.52 \\
\hline $892 \mathrm{~A}-15 \mathrm{X}-1,88-100$ & 116.9 & 223.7 & 0.707094 & 16 & \\
\hline $892 \mathrm{D}-12 \mathrm{X}-3,130-150$ & 123.4 & 260.2 & 0.707043 & 22 & \\
\hline $892 \mathrm{~A}-16 \mathrm{X}-1,34-43$ & 125.8 & 260.2 & 0.707115 & 18 & -0.61 \\
\hline 892 D-13X-CC, $22-24$ & 128.5 & 272.9 & 0.707076 & 15 & -0.56 \\
\hline $892 \mathrm{~A}-17 \mathrm{X}-2,3-13$ & 136.6 & 207.5 & 0.707098 & 18 & -0.68 \\
\hline $892 \mathrm{D}-14 \mathrm{X}-1,115-130$ & 139.2 & 267.2 & & & -0.67 \\
\hline $892 \mathrm{~A}-18 \mathrm{X}-1,115-140$ & 145.8 & 264.9 & 0.707126 & 15 & -0.15 \\
\hline $892 D-15 X-2,135-150$ & 148.9 & 267.2 & 0.707088 & 21 & -0.72 \\
\hline $892 \mathrm{D}-16 \mathrm{X}-3,135-150$ & 161.4 & 267.2 & & & -0.75 \\
\hline $892 \mathrm{D}-16 \mathrm{X}-5,135-150$ & 164.4 & 267.2 & 0.707069 & 18 & \\
\hline $892 \mathrm{~A}-20 \mathrm{X}-2,0-10$ & 165.1 & 278.9 & 0.707109 & 17 & -0.82 \\
\hline
\end{tabular}




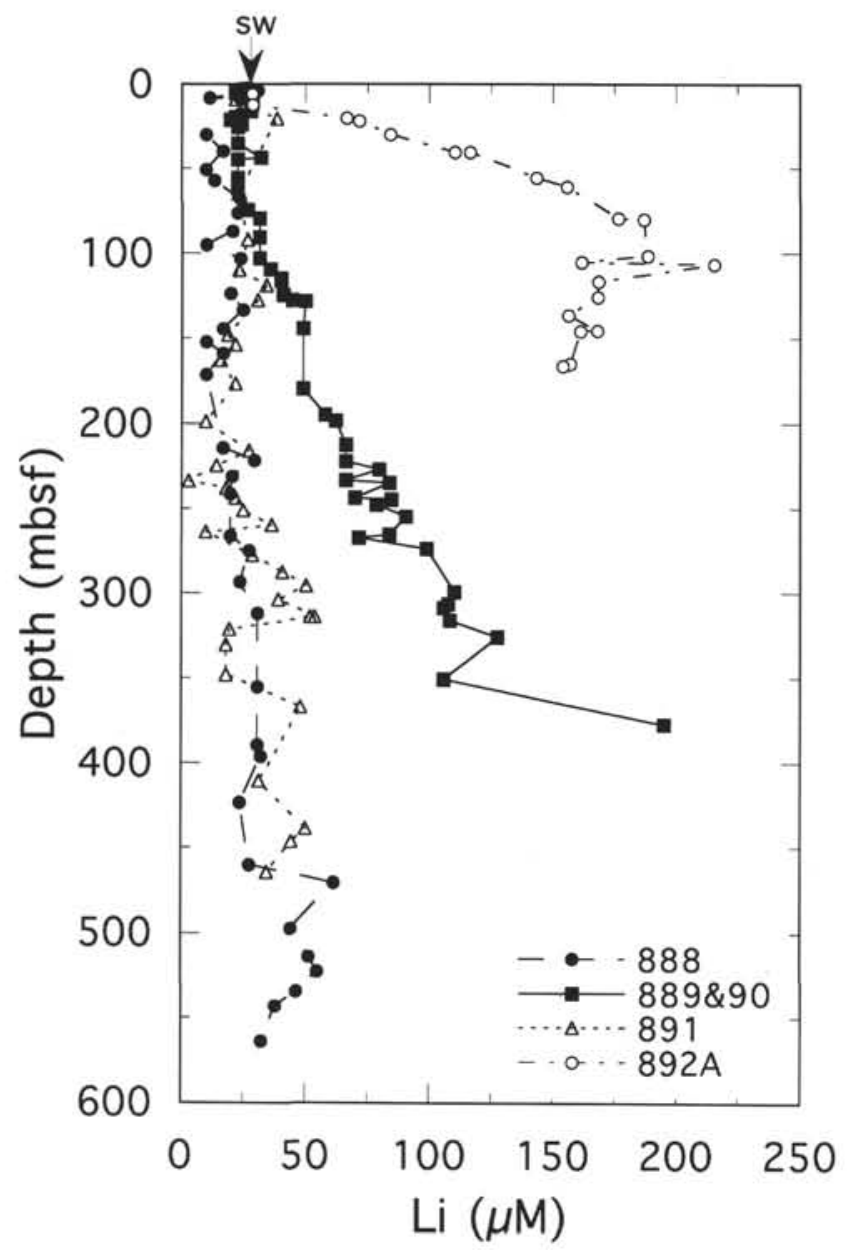

Figure 1. Depth profiles of lithium concentrations in pore fluids from Sites $888,889 / 890,891$, and 892 . The arrow indicates seawater (SW) concentration.

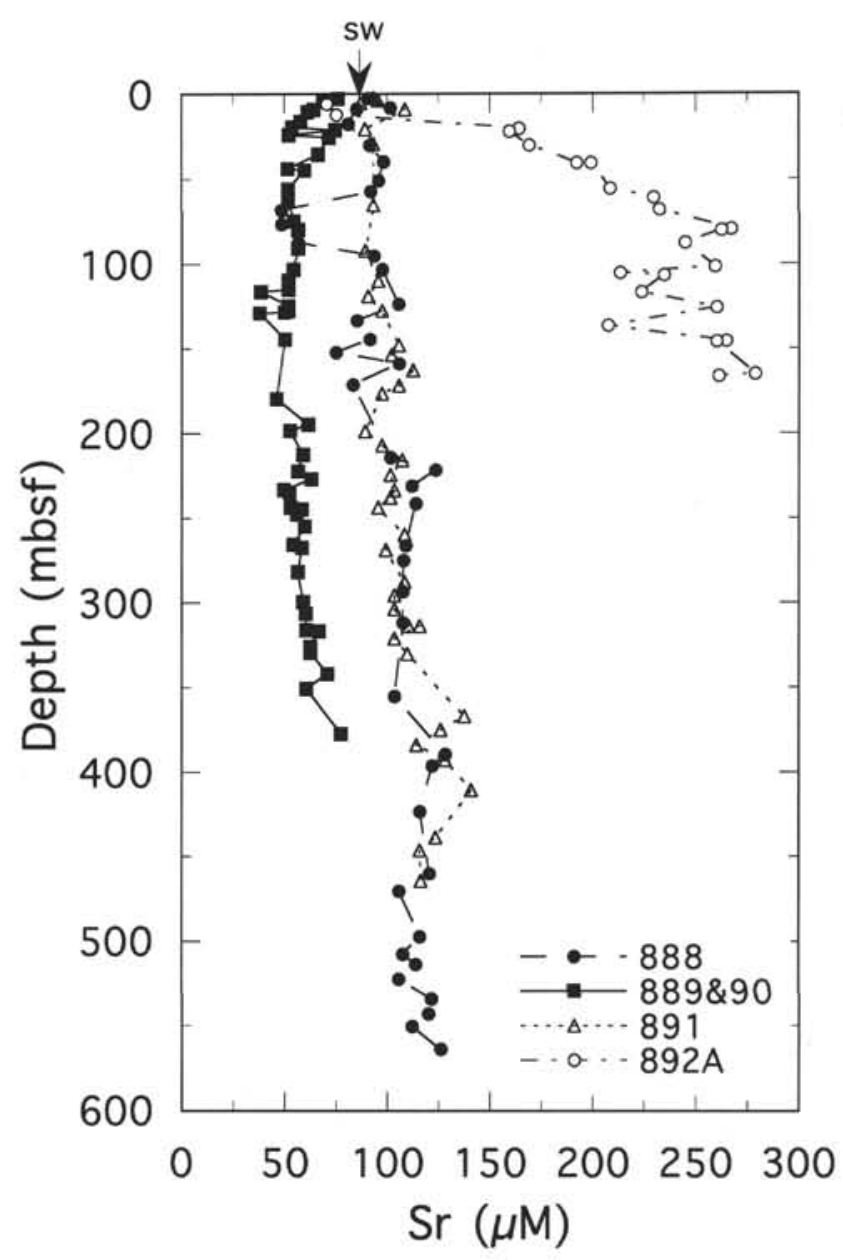

Figure 2. Depth profiles of strontium concentrations in pore fluids from Sites $888,889 / 890,891$, and 892 . The arrow indicates seawater (SW) concentration. 


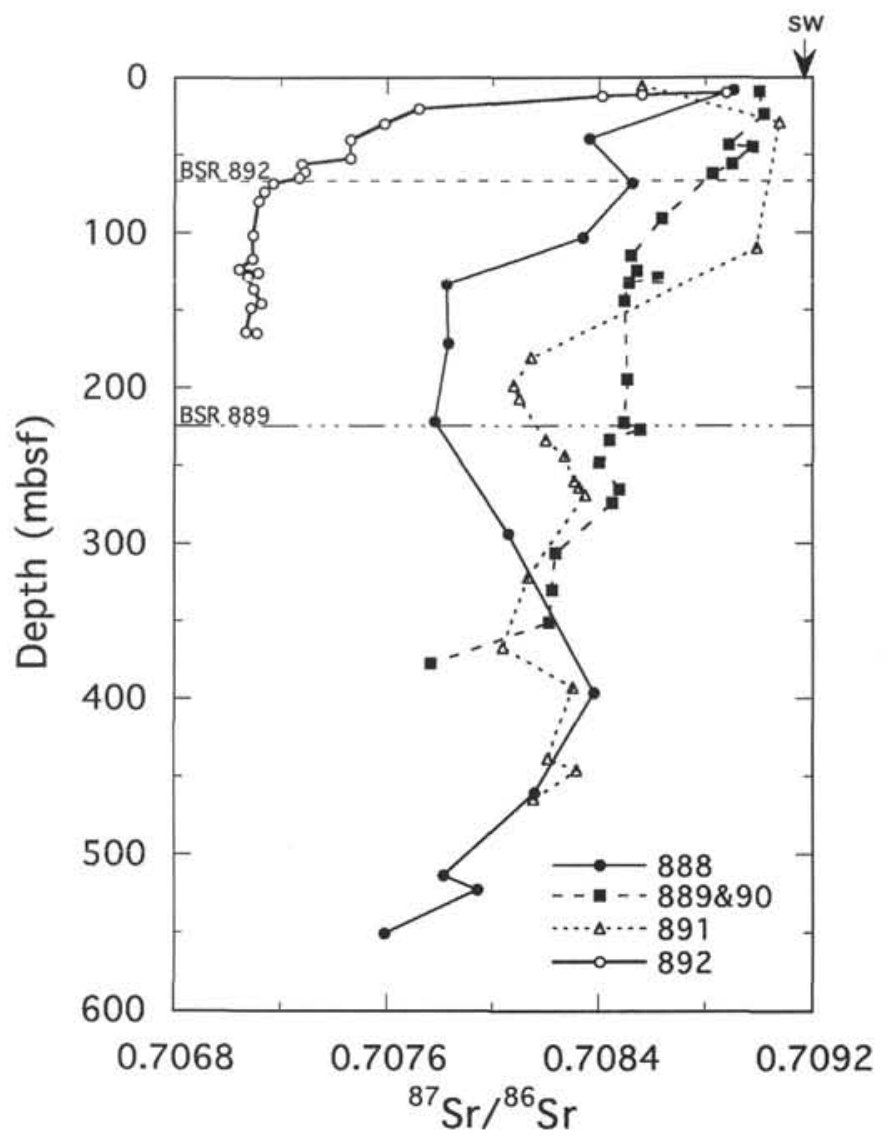

Figure 3. Depth profiles of strontium isotope ratios in pore fluids from Sites $888,889 / 890,891$, and 892 . The arrow indicates seawater (SW) concentration. $\mathrm{BSR}=$ bottom-simulating reflector.

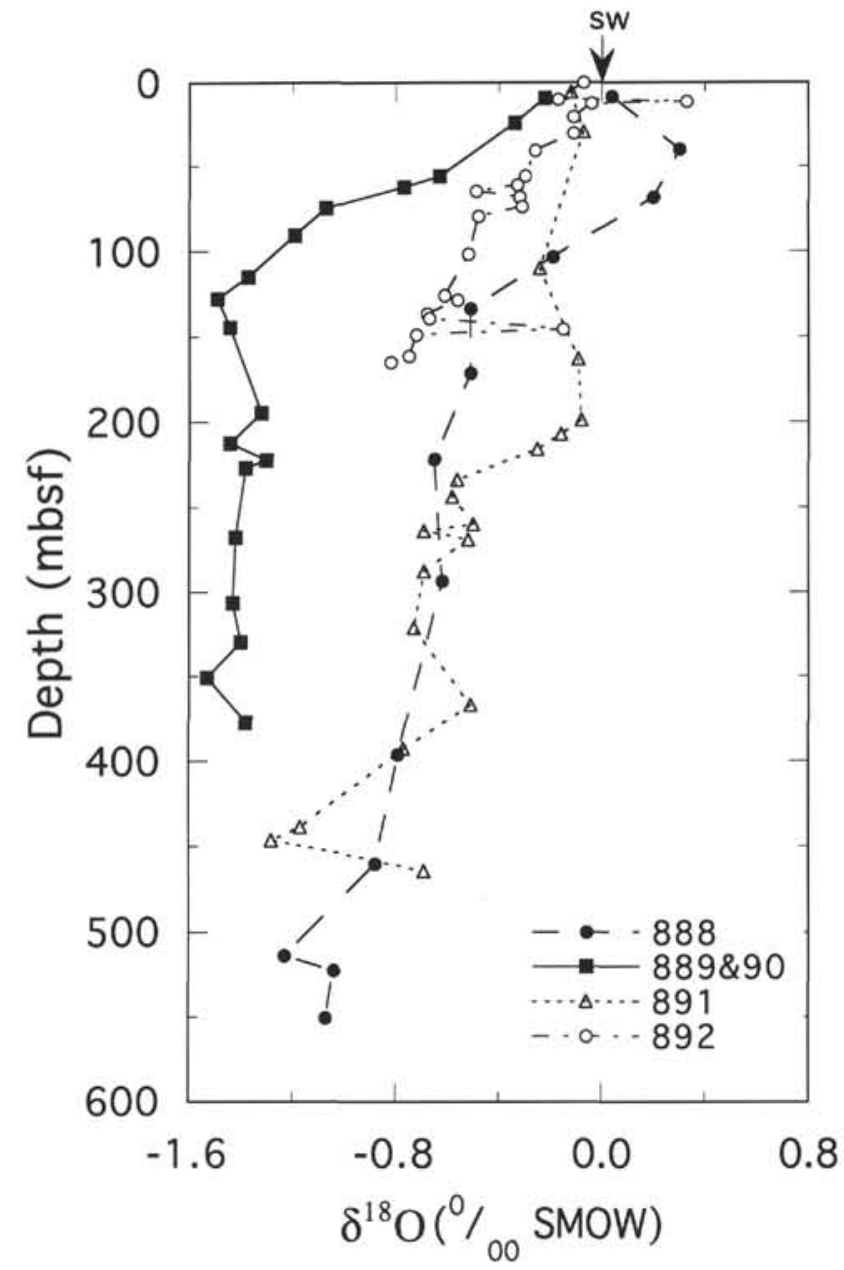

Figure 4. Depth profiles of oxygen isotope ratios in pore fluids from Sites $888,889 / 890,891$, and 892 . The arrow indicates seawater (SW) concentration. 